\title{
Calcifilaxis y enfermedad renal crónica
}

\section{Calciphylaxis and chronic renal disease}

\author{
Jairo Enrique Pérez, Juan Guillermo Vargas, \\ Jorge EnRique EcheVerri, Paula Violeta Rodríguez, \\ Jorge Armando Pulido • Bogota. D.C. (Colombia)
}

\section{Resumen}

Es una vasculopatía caracterizada por calcificación de la capa media de los vasos y proliferación de la íntima de los mismos, asociado a fibrosis y trombosis luminal, llevando a necrosis de los tejidos circundantes, en especial de los tejidos blandos. La fisiopatología de la calcifilaxis está relacionada con múltiples factores, siendo una condición que lleva a la calcificación vascular acelerada y que se ha asociado a una serie de factores de riesgo como la enfermedad renal crónica en terapia de reemplazo y en trasplante renal. Se han relacionado otros factores no urémicos como son anormalidades en el metabolismo del calcio, hiperparatiroidismo primario, estados de hipercoagulabilidad como malignidad, déficit de proteína $\mathrm{C}$ y S, hepatopatía alcohólica, terapia con warfarina, hipoalbuminemia, terapias con calcio y vitamina $\mathrm{D}$, obesidad y producto calcio/fósforo superior a 70 .

A continuación se reporta el caso de una paciente a quien se le documenta un cuadro clínico de calcifilaxis, describiendo la presentación clínica de esta patología, los factores de riesgo identificados y las complicaciones presentadas durante su atención (Acta Med Colomb 2011; 36: 149-152).

Palabras clave: vasculopatía, calcifilaxis, arteriolopatia calcificante urémica, enfermedad renal crónica, hiperparatiroidismo primario.

\begin{abstract}
Calciphylaxis is a vasculopathy characterized by calcification of the tunica media and proliferation of the tunica intima of blood vessels, in association with fibrosis and thrombosis of the lumen, which lead to necrosis of surrounding (especially soft) tissues. The pathophysiology of calciphylaxis is related with multiple factors. This condition leads to accelerated vascular calcification and has been associated with a number of risk factors, such as chronic renal disease, kidney replacement therapy, and renal transplantation. It has also been related with other, non-uremic, factors, such as calcium deregulation, primary hyperparathyroidism, hypercoagulability states (such as malignancy and deficiency of proteins $\mathrm{C}$ and S), alcoholic hepatopathy, warfarin therapy, hypoalbuminemia, therapy with calcium and vitamin $\mathrm{D}$, obesity, and a Calcium/Phosphorus product above 70 .

We report the case of a patient with calciphylaxis by describing the clinical presentation, the associated risk factors, and the complications seen during the care of the patient (Acta Med Colomb 2011; 36: 149-152).

Key words: vasculopathy, calciphylaxis, calcific uremic arteriolopathy, renal chronic disease, primary hyperparatiroidism.
\end{abstract}

Dr Jairo Enrique Pérez: Especialista en Medicina Interna, Epidemiólogo Clinico. Hospital Militar Central; Dr. Juan Guillermo Vargas: Especialista en Medicina Interna, Nefrología y Epidemiología Clínica. Director Médico Unidad Renal Hospital Universitario Mayor. Coordinador Programa de Emergencia Renal RTS. Profesor Facultad de Medicina Universidad el Rosario; Dr Jorge Enrique Echeverri: Especialista en Medicina Interna, Nefrología y Cuidado Intensivo. Coordinador Académico del Posgrado de Nefrología Universidad Militar Nueva Granada. Jefe Servicio de Nefrología Hospital Militar Central. Coordinador Programa de Nefrología Crítica Hospital Universitario San Ignacio. Profesor Facultad de Medicina Universidad Militar Nueva Granada y Pontificia Universidad Javeriana; Dra. Paula Violeta Rodríguez: Residente Medicina Interna, Hospital Militar Central. Universidad Militar Nueva Granada; Dr Jorge Armando Pulido, Residente Medicina Interna. Hospital Militar Central. Bogotá, D.C. (Colombia)

Correspondencia. Dr. Juan Guillermo Vargas. Bogotá, D.C. (Colombia).

E-mail: juanguillermovargas@yahoo.com Recibido: 13/II/2011 Aceptado: 03/VIII/2011

\section{Caso clínico}

Mujer de 41 años, con antecedente de enfermedad renal crónica de etiología hipertensiva, en terapia de reemplazo renal modalidad diálisis peritoneal automatizada durante los últimos diez años. Consulta por cuadro clínico de dos meses de evolución consistente en aparición de lesiones irregulares, necróticas en miembros inferiores, dolorosas que progresan en extensión. Al ingreso presenta tensión arterial de 130/70 $\mathrm{mmHg}$, frecuencia cardiaca de 110 latidos por minuto, frecuencia respiratoria 24 respiraciones por minuto, temperatura $37.2^{\circ} \mathrm{C}$, con presencia de lesiones tipo placa con bordes purpúricos y centro necrótico, calor local y asociadas a costras serohemáticas a nivel de tercio inferior de muslos y cara posterior de piernas (Figuras 1 y 2).

En los paraclínicos de ingreso, el hemograma evidencia un recuento de 15310 leucocitos, neutrófilos de $86 \%$, linfocitos de $7.9 \%$, hemoglobina de $6.2 \mathrm{mg} / \mathrm{dL}$, plaquetas 484.000 , creatinina $9.5 \mathrm{mg} / \mathrm{dL}$, nitrógeno ureico en sangre $101 \mathrm{mg} / \mathrm{dL}$, calcio total $7.8 \mathrm{mg} / \mathrm{dL}$ y fósforo de $10.7 \mathrm{mg} / \mathrm{dL}$. 
Con el fin de valuar calcificación aórtica y de vasculatura periférica se realiza radiografía de tórax y radiografía de miembros inferiores que evidencian calcificación de distintos tractos vasculares (Figuras 3 y 4 ).

Se realiza un diagnóstico inicial de infección de tejidos blandos, empezando terapia antimicrobiana, analgésicos y manejo local de las lesiones con curaciones y desbridamiento de las mismas. Se complementan estudios con hormona paratiroidea intacta, cuyo valor reporta $1700 \mathrm{pg} / \mathrm{ml}$. Se ajusta terapia dialítica iniciando manejo médico para hiperfosfatemia.

Biopsia cutánea con evidencia en los cortes histológicos de depósitos granulares finos basofílicos compatibles con depósitos de calcio, necrosis isquémica de glándulas ecrinas, trombos de fibrinas en vasos pequeños y escasos neutrófilos perivasculares (Figura 5 y 6 ).

Se considera hiperparatiroroidismo secundario con calcifilaxis asociada e indicación de paratiroidectomía una vez se resolviera el proceso infeccioso. La paciente presenta
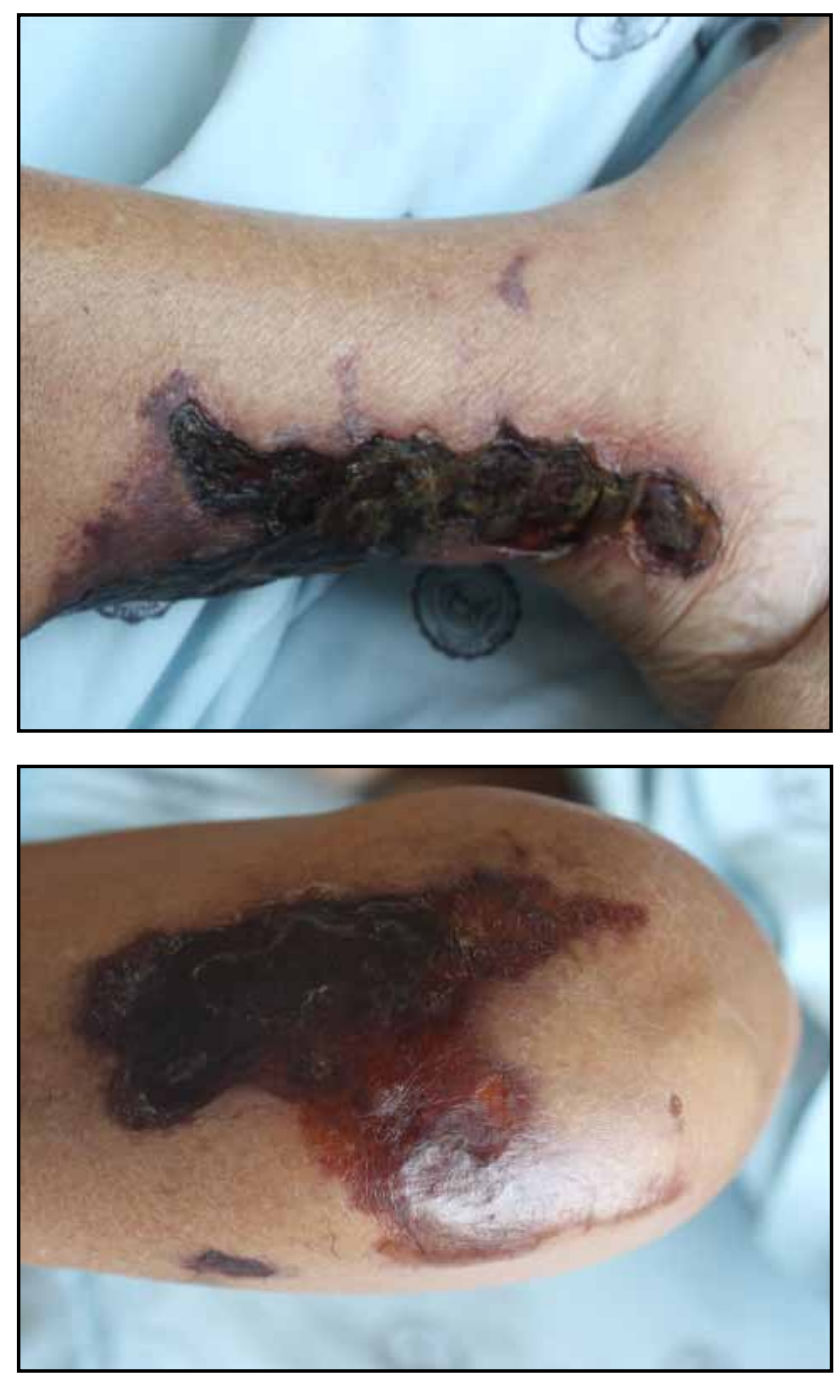

Figuras 1 y 2. Lesiones en piel de miembros inferiores. evolución tórpida con progresión a sepsis severa, disfunción multiorgánica sin respuesta a tratamiento médico y posterior muerte.

\section{Discusión}

La calcifilaxis es una entidad reportada por primera vez por Brian y Whites en 1898, y posteriormente descrita por Selye a través de su modelo experimental con ratas nefrectomizadas. Desde entonces es conocida como arteriolopatía calcificante urémica o calcifilaxis (inflamación "anafiláctica" de los vasos seguida de calcificación de los mismos) (1), de cuya fisiopatología se han planteado un sinnúmero de hipótesis aun sin demostrar.

En el caso descrito se pueden observar algunas de las características más frecuentes de los pacientes con calcifilaxis, como es su presentación en pacientes con enfermedad renal crónica avanzada y en soporte dialítico de larga data. La serie de casos reportada por Angelis y cols, describe 242 casos de pacientes en terapia de reemplazo renal, reportando
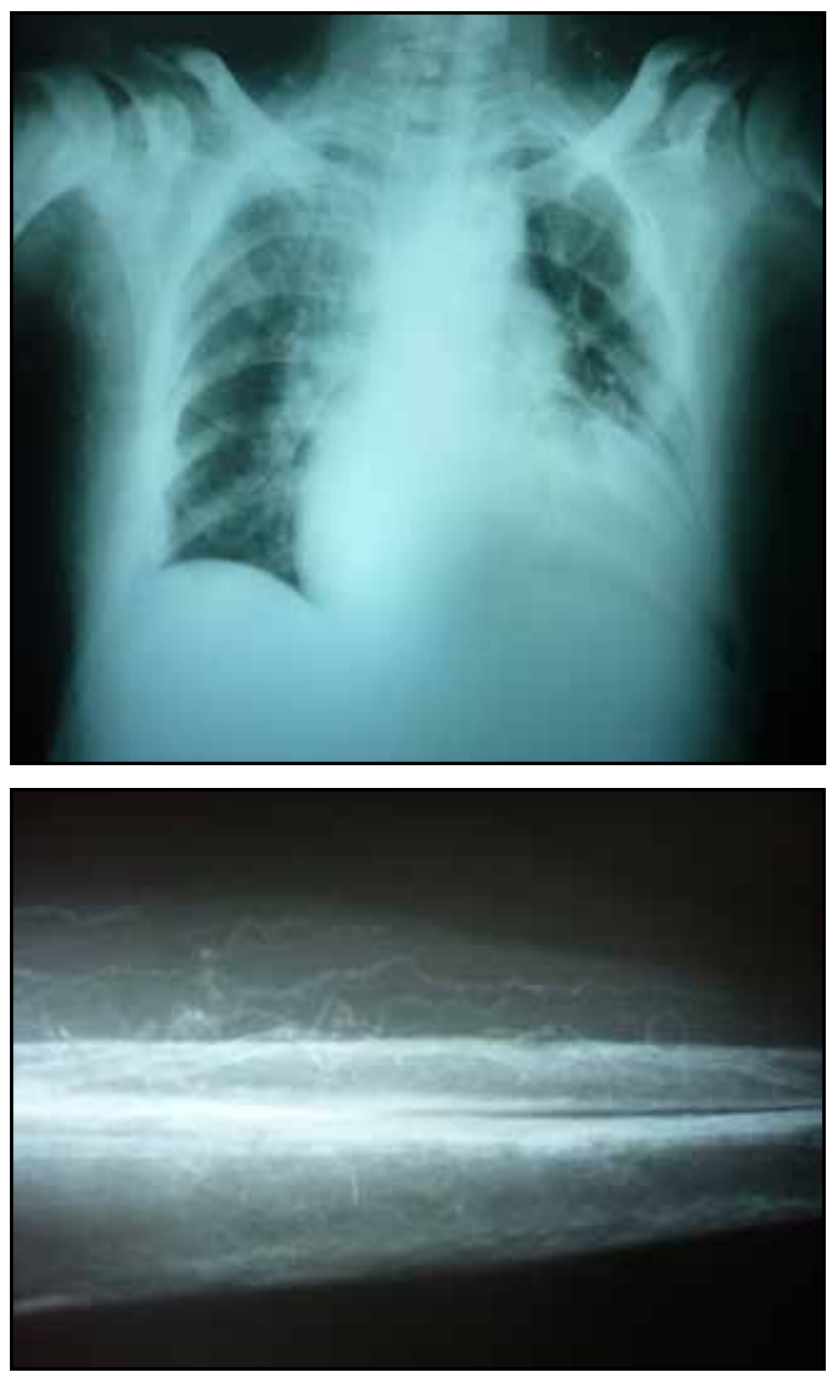

Figuras 3 y 4. Imágenes de calcificación de tractos vasculares en tórax y en miembros inferiores 

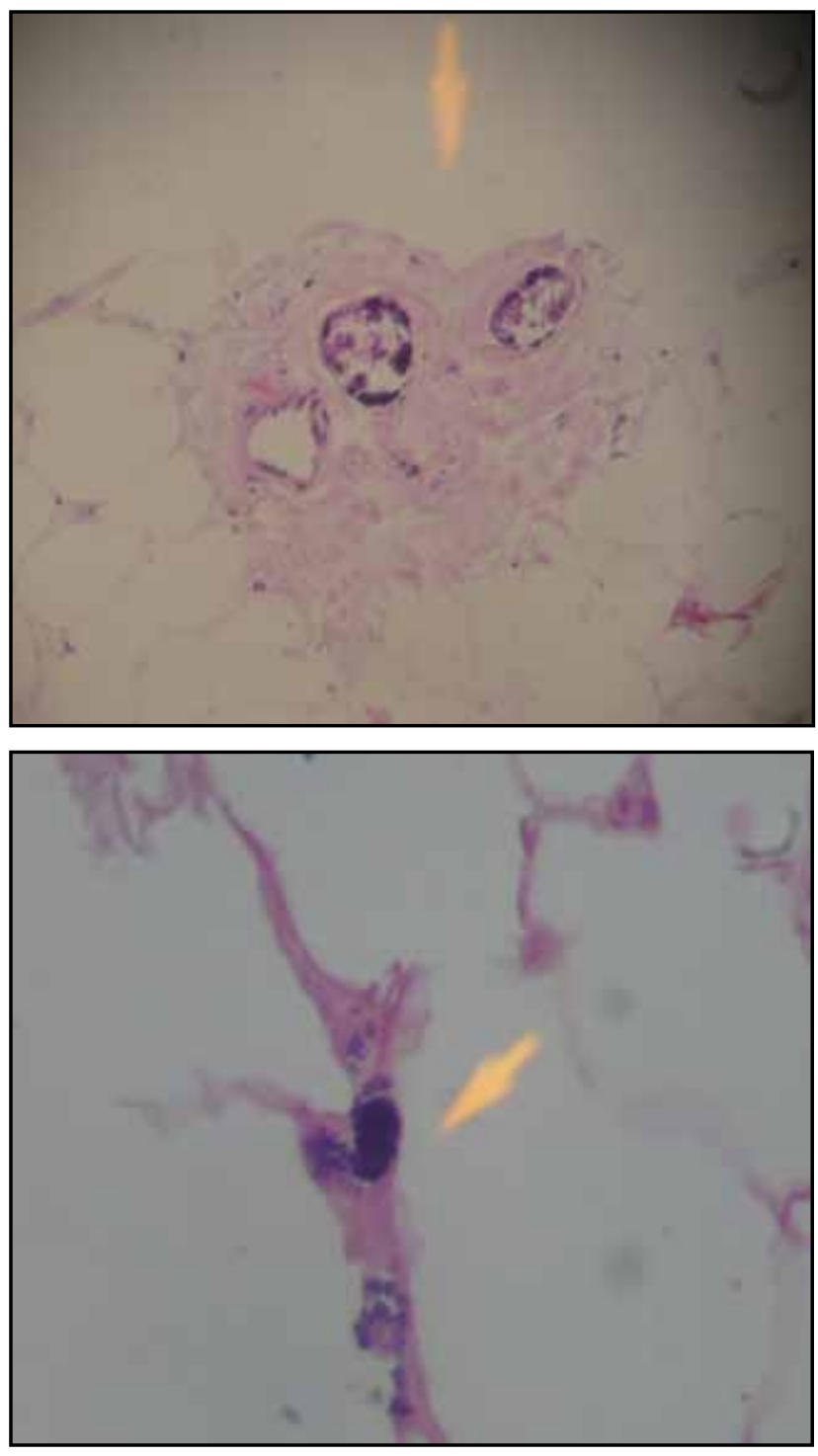

Figuras 5 y 6. Histopatológia con evidencia de calcificación de pared vascular y formación de trombos intraluminales

una incidencia de $4 \%$ de calcifilaxis, en su mayoría de sexo femenino, con presencia de úlceras necróticas en miembros inferiores secundarias a calcificación de pequeños vasos y necrosis de tisular con desenlace fatal, como lo reportado en el caso actual (2). Existe un reto diagnóstico en estos pacientes, siendo la biopsia de las lesiones el patrón de oro confirmatorio.

Acerca de la patogenia de la calcificación de los vasos, se han descrito múltiples mecanismos, los cuales proponen una alteración en la homeostasis entre factores inductores e inhibidores del depósito de calcio a nivel de la pared vascular con posterior precipitación de éste. Adicionalmente existe una expresión de proteínas óseas, complejos de nucleación ósea y activación de genes que sintetizan proteínas como osteopontinas y osteocalcina, que alteran el metabolismo del calcio, perpetuando este ciclo.Así mismo han sido descritos algunos transportadores como el Pit1, que con el incremento de fosfato sérico tienen un efecto similar, predisponiendo al depósito vascular de calcio (3).

Se han reportado factores que inhiben el depósito de calcio a nivel extraóseo, la osteoprotegerina protege a las células endoteliales de la apoptosis inducida por la inflamación, inhibiendo el ligando del factor nuclear Kappa Beta (RANK) que está íntimamente relacionado con la mineralización ósea y periférica. La proteína Gla de la matriz ósea inhibe la acción de multiples proteínas óseas alterando la capacidad de unión y depósito de calcio a la matriz ósea extracelular (4). El fetuin A es un importante inhibidor circulante de la calcificación y se disminuye durante las fases inflamatorias persistentes. Datos serológicos en pacientes con tiempos prolongados de diálisis y bajas concentraciones del fetuin $\mathrm{A}$, han mostrado precipitación vascular de calcio y fósforo. Aparentemente este factor influencia en la resolución de la inflamación, modulando la fagocitosis de células apoptóticas por macrófagos (5).

El papel de la hormona partiroidea (PTH) aún no está muy claro. Bogdonoff y cols., (6) reportaron en la necropsia de dos pacientes con calcifilaxis la presencia de adenomas paratiroideos e historia previa de niveles séricos elevados de PTH. Se ha planteado que la elevación persistente de los niveles de PTH por encima de $800 \mathrm{pg} / \mathrm{ml}$ se relaciona con aumento del riesgo de calcificación vascular, y la falta de respuesta clínica rápida al manejo médico indica realización de paratiroidectomía. Sin embargo, la relación de los niveles elevados de PTH con la fisiopatología de la enfermedad está aún por establecer (7).

La calcifilaxis es una enfermedad potencialmente fatal, con una tasa de mortalidad a 12 meses cercana a $80 \%$ (8). Los esquemas de tratamiento han evolucionado a medida que se ha clarificado el papel fisiopatológico del metabolismo calcio-fósforo en la enfermedad. Se han propuesto diferentes alternativas terapéuticas orientadas a la disminución de la absorción intestinal de fosfatos, dentro de ellas la dieta y el uso de quelantes iónicos y no iónicos de fósforo, sin embargo el uso de éstos no ha podido dar un impacto claro en disminución de mortalidad (11). El uso de calciomiméticos se ha propuesto con el objetivo de disminuir la sensibilidad a la PTH y aumentar la sensibilidad de los canales de calcio. Los esteroides se han propuesto con el fin de disminuir la respuesta inflamatoria asociada a la vasculopatía y los bifosfonatos (pamidronato y etidronato) con el fin de aumentar la producción de osteoprotegerina y con ello disminuir la calcificación intravascular $(9,10)$. El tiosulfato de sodio se ha asociado a cierta mejoría en algunas series de casos, sin embargo, su mecanismo de acción aún no es claro y sus efectos adversos aún superan el beneficio sin modificación del pronóstico en los pacientes $(11,12)$. Otras intervenciones, como el uso de heparinas en estados hipercoagulables (13), el uso de cámara hiperbárica (14), la realización de paratiroidectomía (15) y el desbridamiento quirúrgico profiláctico de las lesiones para evitar infecciones, han sido reportados 
sin impactar en la historia natural de la enfermedad. Hasta el momento con las líneas de tratamiento actuales, el pronóstico de la calcifilaxis continúa siendo pobre con una mortalidad elevada, siendo la sepsis la causa más frecuente de la muerte en estos pacientes como ocurrió en el caso presentado (16).

\section{Referencias}

1. Seyle H. Calciphylaxis. Chicago, IL. University of Chicago Press. 1962.

2. Angelis M, Wong LL, Myers SA, Wong LM. Calciphylaxis in patients on hemodialysis: a prevalence study. Surgery 1997; 122: 1083-9.

3. Proudfoot S, Shanahan CM. Molecular Mechanisms mediating vascular calcification role of matrix Gla - Protein. Nephrology 2.006; 3: 1139-43.

4. Boyce B, Xing L. Biology of RANK, RANKL, and Osteoprotegerin. Arthritis Research \& Therapy 2007; 9(Suppl 1): S1.

5. Mehrotra R.Emergin Role for Fetuin - A as contributor to morbidity and mortality in chronic kidney disease. Kidney International 2007; 15: 2959-64.

6. Bogdonoff MD, Engel FL, White JE, Woods AH. Hyperparathyroidism. Am J Med 1956; 21: 583-95.

7. Girotto JA, Harmon JW, Ratner LE, et al. Parathyroidectomy promotes wound healing and prolongs survival in patients with calciphylaxis from secondary hyperparathyroidism. Surgery 2001; 130(4): 645 .

8. Nigwekar SU, Wolf M, Sterns RH, Hix JK. Calciphylaxis from nonuremic causes: a systematic review. Clin J Am Soc Nephrol 2008; 3: 1139-43.

9. Arch-Ferrer J, Beenken S, Therapy for calciphylaxis: An outcome analisys, Surgery 2003; 134(6): 941-4.

10. Wilmer WA, et al. Calciphylaxis: emerging concepts in prevention, diagnosis and treatment. Semin Dial 2002; 15(3): 172:86

11. Price PA, Faus SA, Williamson MK. Bisphosphonates alendronate and ibandronate inhibit artery calcification at doses comparable to those that inhibit bone resorption. Arterioscler Thromb Vasc Biol 2001 May; 21(5): 817-24.

12. Meissner M, Kaufmann R, Gille J. Sodium thiosulphate: a new way of treatment for calciphylaxis?. Dermatology 2007; 214(4): 278-82.

13. Cozzolino M, Brandenburg V. Warfarin: to use or not to use in chronic kidney disease patients? J Nephrol 2010; 23: 648-52.

14. Rogers NM, Chang SH, Teubner DJ, Coates PT. Hyperbaric oxygen as effective adjuvant therapy in the treatmentof distal calcific uraemic arteriolopathy. Nephrol Dial Transplant 2008; 1: 244-9.

15. Girotto JA, Harmon JW, Ratner LE, et al. Parathyroidectomy promotes wound healing and prolongs survival in patients with calciphylaxis from secondary hyperparathyroidism. Surgery 2001; 130: 645-50.

16. Duth QY, Lim RC, Clarck OH. Calciphylaxis in secondary hyperparatiroidism. Arch Surg 1991; 126: 1218-9. 